\title{
QUAND LA FEINTE ET L'ENVIE S'ATTAQUENT AU SACRÉ : QUELQUES NOTES SUR LES REPRÉSENTATIONS DISSONANTES DE L'ABBAYE DE GÊNES-SUR-MER DANS LE MONIAGE GUILLAUME I
}

\author{
Ján ŽIVČÁK \\ Université de Prešov, Prešov
}

\begin{abstract}
En): The present study aims to analyse the dissonant representations of the Gênes-surMer abbey in the Moniage Guillaume I (William in the monastery I), a 12th-century Old French heroic epic. The singularity of this paradoxical locus resides in the discrepancies that the text establishes between two mental projections doubling, within the plot, the physical modality of the monastery. Whilst the mental projection of the abbey shaped by the fictional conscience of the monastic community shows several spatial attributes of a sacred locus (a cosmos), in the eyes of the narrator, the monastery is equated with a moral chaos. This study attempts to analyse the "conflict" between the narrator's perspective and the perspective of the monks which underlies the whole plot and culminates in the mirror effect that the text establishes between the space of the abbey and that of the dreadful Beaucler forest, and in the scene of (self)destruction of the space of the monastery, representing a consequence of the "avalanche of perfidy" which covers the abbey in the second half of the epic.
\end{abstract}

Keywords (En): Moniage Guillaume I (William in the Monastery I); Old French epic; monastery in literature; sacred space and profane space; medieval narration.

Mots-clés (Fr): Moniage Guillaume I ; chanson de geste ; monastère en littérature ; espace sacré et espace profane ; narration au Moyen Âge.

\section{Introduction}

Durant le Moyen Âge central, peu d'édifices pouvaient jouir d'un statut aussi exceptionnel que les monastères. Aux yeux du peuple, ces constructions complexes de grande superficie et de remarquable richesse architecturale (notamment dans le cas de la famille bénédictine) appartenaient presque à un monde à part, à un monde sacré où l'homme, dépourvu de toute relation avec l'univers profane, s'unit intimement à son Créateur ${ }^{1}$. Cette façon de concevoir les espaces monastiques laissa des empreintes dans la littérature : les monastères ou les abbayes occupent une place éminente dans les continuums spatiaux ${ }^{2}$ de nombreux textes médiévaux tant d'origine religieuse que d'origine laïque. Dans le Moniage Guillaume I, une chanson de geste incomplète datant de la moitié du XII ${ }^{\mathrm{e}}$ siècle et qui constituera le corpus de notre étude, l'espace monastique en tant que partie intégrante du monde fictionnel ${ }^{3}$ fait son apparition dès les premières laisses ${ }^{4}$. Le marquis Guillaume (dit Guillaume d'Orange ou Willame al curb niés), le protagoniste de l'épopée ayant

\footnotetext{
${ }^{1}$ Pour plus d'informations sur le monasticisme médiéval, voir, par exemple, MelviLle (2016).

2 Terme de Youri Lotman désignant la totalité des espaces figurant dans un texte littéraire et les rapports existant entre les différentes modalités spatiales. Voir LOTMAN (1973).

${ }^{3}$ Pour plus de détails sur le contenu du terme « monde fictionnel », voir DOLEŽEL (1998).

${ }^{4}$ Il est question de la soi-disant « rédaction courte» du Moniage Guillaume. Toutes les citations de l'étude se référeront à l'édition de Wilhelm Cloetta, présentant une version de 934 vers majoritairement décasyllabiques. Pour davantage de renseignements sur le Moniage Guillaume I (les origines, la date de composition, l'étude des manuscrits, etc.), voir ClOETTA (1911).
} 
perdu son épouse Guibourc, décide de quitter sa forteresse et d'entrer en religion. À la recommandation d'un ange qui lui apparaît à la suite des funérailles, il se met en route pour Gênes-sur-Mer (Genevois sour mer) où il reçoit la tonsure dans une abbaye bénédictine.

Or, contrairement aux éventuelles attentes des lecteurs ou des auditeurs, l'abbaye de Gênes-sur-Mer n'incarne en aucun cas un locus paisible et imprégné du sacré. Bien au contraire, sa représentation (ou plutôt ses représentations) épique(s) se montre(nt) inquiétante(s) et dissonante(s): les membres de la communauté religieuse, mécontents du comportement et du caractère quelque peu grossier du protagoniste, décident de trahir, peu après la tonsure de ce dernier, le commandement biblique de l'amour envers le prochain. Afin de dévoiler les racines de ce paradoxe, nous tâcherons d'élucider la configuration épique de cet étrange monastère. En nous appuyant sur les axiomes théoriques de Mircea ELIADE (1965), résumés dans l'ouvrage Le sacré et le profane, et sur la terminologie de Xénia Činčurová, théoricienne slovaque de l'espace littéraire, nous nous efforcerons d'identifier les attributs spatiaux ${ }^{5}$ des représentations textuelles de l'abbaye en question, et de dévoiler ainsi sa sémantique ${ }^{6}$ et sa symbolique ${ }^{7}$.

Cependant, pour rendre plus transparentes nos analyses et interprétations, consacrons encore quelques lignes à l'explication du titre de la présente étude. Ce n'est pas un hasard si au lieu de parler d'une représentation, nous évoquons les représentations au pluriel. En effet, dans le tissu narratif de l'épopée, trois représentations différentes se rapportent à l'espace du monastère de Gênes-sur-Mer. Tout d'abord, le texte expose des descriptions factuelles (topographies) de la modalité physique ou architecturale de l'abbaye. Le lecteur ou l'auditeur apprend que le monastère épique revêt la forme d'un grand complexe avec un aménagement intérieur riche et diversifié. Dans le cloître s'étendant derrière les murs de l'édifice, sont localisés les cellules des moines, un dortoir et un chapitre. La relative autonomie économique du monastère est assurée par la cuisine, le cellier et l'étable qui se situent, eux aussi, à l'intérieur des murailles. Au milieu du complexe se tient l'église, centre spirituel de la communauté. L'ensemble du locus se constitue comme un espace parfaitement clos, protégé par des murailles résistantes et difficilement pénétrables. Pour parvenir dans le cloître, il est nécessaire de franchir une porte robuste et solide, munie de lourdes barrières. Mais hormis les topographies objectales du complexe monastique, le texte intègre deux projections mentales de la localité concernée. La première appartient à la conscience fictionnelle du narrateur, la seconde est le fruit de la conscience collective des moines résidant au monastère. Comme en témoignera la suite de l'étude, dans le système de valeurs propres au texte, la première s'oppose à la seconde de manière radicale. Cette discordance (qui entraîne en même temps une dissonance entre l'optique du

\footnotetext{
${ }^{5}$ Terme emprunté à Xénia Činčurová, dénotant l'ensemble de qualités qui déterminent comment les personnages et le narrateur perçoivent un espace littéraire. Voir Č́INČUROVÁ (2004).

${ }^{6}$ Terme de Xénia Činčurová. Pour déterminer la sémantique d'un espace littéraire, il faut définir ce que ce dernier représente pour les personnages ou pour le narrateur. Voir ČINČUROVÁ (2004).

${ }^{7}$ Terme de Xénia Činčurová. La symbolique d'un espace littéraire correspond au sens le plus abstrait (philosophique ou métaphysique) que l'auteur attribue à tel ou tel élément du continuum spatial. Voir ČINČUROVÁ (2004).
} 
narrateur et l'optique des personnages) est tellement significative que l'ensemble du récit (dans le sens genettien du terme) ${ }^{8}$ semble figurer une lutte métaphorique entre les positions axiologiques du narrateur et celles des personnages. Le conflit intradiégétique qui se joue entre les moines et le protagoniste au niveau de l'histoire est ainsi accompagné d'un conflit extradiégétique entre les entités narratives, dont l'objet est la position de la représentation épique du monastère sur l'axe « espace sacré - espace profane $»^{9}$.

Pour mieux démontrer les stratégies des différentes entités narratives dont les discours façonnent l'image épique de la localité analysée, l'organisation de notre argumentation sera assimilée à l'organisation du conflit métaphorique qui vient d'être évoqué. Seront abordés en premier lieu les attributs spatiaux de la projection mentale de l'abbaye telle qu'elle se dresse dans la conscience collective des moines. En deuxième lieu, nous traiterons la catégorie du narrateur, les aspects axiologiques de sa prise de position et sa vision singulière du locus analysé. Enfin, en troisième lieu, nous tâcherons de dévoiler les mécanismes de la lutte métaphorique que l'auteur épique se plaît à créer entre les deux conceptions, et nous expliquerons les causes et les conséquences du dénouement de ce drôle de conflit.

\section{L'abbaye de Gênes-sur-Mer vue par la communauté monastique}

Le jugement que les moines portent sur leur espace vital découle de leur attitude envers lui. Dès l'exposition de la chanson de geste, il paraît évident que la communauté monastique de Gênes-sur-Mer vit dans une harmonie parfaite avec son lieu de résidence. Les moines sont métaphoriquement parlant «ancrés dans les murs » de l'abbaye et le monastère représente pour eux un foyer ressenti comme leur, un véritable $\operatorname{cosmos}^{10}$. Le lecteur ou l'auditeur découvre ces profondes relations de sympathie notamment grâce aux répliques (c'est-à-dire grâce aux morceaux de discours direct) prononcées par différents moines et qui mettent en valeur (quoique souvent implicitement) le caractère fin et distingué du locus analysé. Il n'est donc pas étonnant que la projection mentale du monastère surgissant dans la conscience des moines partage un nombre significatif des attributs que Mircea Eliade assigne aux espaces sacrés ${ }^{11}$.

Premièrement, l'ensemble de la communauté monastique perçoit le complexe de l'abbaye comme un lieu paisible, harmonieux, prévisible et parfaitement organisé. Les jours y coulent de manière monotone et symétrique et les règles et coutumes qui gèrent la vie monastique ne changent (presque) jamais. Comme ce fut

\footnotetext{
${ }^{8}$ Dans la conception narratologique de Gérard Genette, le terme «récit» désigne la configuration finale d'une histoire, c'est-à-dire d'une série d'évènements relatés par une instance narrative et qui constitue l'intrigue d'un texte littéraire. Voir GeNETTE (1972).

9 Pour plus de détails sur le contenu des termes «(niveau) extradiégétique » et "(niveau) intradiégétique », voir GENETTE (1972).

${ }^{10}$ Dans Le sacré et le profane, Mircea Eliade (1965) utilise le terme de «cosmos » pour qualifier un espace (parfaitement) sacré. Le terme «chaos » est employé, au contraire, pour évoquer un espace (parfaitement) profane.

${ }^{11}$ Dans Le sacré et le profane, Mircea ELIADE (1965) prête aux espaces sacrés dans leur forme pure les attributs suivants : organisé, réel, constituant le Centre du Monde, formé par des dieux, ouvert vers le haut, clos, ressenti comme sien et consacré par un sacrifice sanglant.
} 
- et comme c'est même aujourd'hui - le cas dans les monastères contemplatifs, les activités des moines sont rythmées par la prière du bréviaire. La nature strictement organisée de l'espace de l'abbaye vue par la communauté religieuse se laisse entendre notamment dans les passages où l'abbé présente à Guillaume, nouvellement arrivé au monastère, les principes de la vie dans les ordres :

\author{
Sire Guillaume, proudom estes et sire ; \\ Si m'äit Dex, nous t'aprendrons a lire \\ Vostre sautier et a chanter matines \\ Et tierce et none et vespres et conplie. \\ Quant serés prestres, si lirés l'evangile, \\ Et si chanterés messe. \\ (Le Moniage Guillaume 1906 : 6, v. 136-141)
}

Deuxièmement, au regard des moines, l'abbaye représente un espace absolument rassurant. L'intérieur du complexe monastique (à l'instar des territoires sacrés des sociétés archaïques analysés par Mircea Eliade) est strictement isolé du monde ordinaire, entouré par des murailles (servant également de protection contre les invasions sarrasines) et fermé par une grosse porte en bois que seule une force surhumaine arriverait à rompre. Les murs et la porte figurent au sein du continuum spatial comme manifestations matérielles de la frontière symbolique séparant l'espace de l'abbaye, jugé souverainement sacré, des territoires externes, considérés (à l'instar des localités profanes des sociétés primitives étudiées par Eliade) comme dangereux, vicieux et instables. La stabilité interne du complexe monastique est encore amplifiée par la présence d'une église, incarnant le centre inébranlable du cloître, et abritant l'Eucharistie que la théologie catholique identifie, entre autres, à la source de vie de tout chrétien ${ }^{12}$. Il est à noter que dans le Moniage Guillaume I, les moines n'osent jamais quitter l'espace du monastère (le seul personnage qui quitte temporairement le cloître est Guillaume, envoyé en ville par l'abbé pour acheter des poissons) et n'acceptent aucune visite de l'extérieur (la seule exception étant, de nouveau, le marquis Guillaume au moment où il entre au noviciat). Aussi, le texte ne contient aucune mention de fidèles laïcs qui participeraient aux célébrations liturgiques à l'église monastique.

Enfin, troisièmement, dans la conscience fictionnelle des moines, l'abbaye s'identifie à un locus de haute culture, d'érudition et de bonnes manières (que la communauté religieuse apprécie). Les moines résidant dans le monastère savent tous lire, écrire et chanter. Bref, la projection mentale de l'espace du monastère qui se dresse dans la conscience collective de la communauté monastique prend la forme d'un locus parfaitement sacré que les religieux cultivent et considèrent comme leur.

Il n'est donc guère surprenant que la tonsure de Guillaume soit perçue par ses confrères comme un moment perturbateur ${ }^{13}$. Guillaume est un personnage grossier, un bellator natus ayant passé toute sa vie épique au service du roi Louis. Bien qu'il

\footnotetext{
${ }^{12}$ Pour davantage de renseignements sur les rapports entre le sacrement de l'Eucharistie et la vie (au sens large du terme) dans la théologie catholique, voir, par exemple, TKÁČ (2012).

${ }^{13}$ Plusieurs assertions dans le paragraphe s'inspirent des idées d'André MoISAN (1995) et de Pierre MANEN (2014).
} 
s'efforce de s'adapter aux coutumes et au rythme de la vie dans les ordres, il reste, tout au long du Moniage Guillaume I, soumis davantage aux règles de la vie guerrière qu'à la règle bénédictine, et incarne ainsi, aux yeux de la communauté monastique, un élément étranger et inquiétant, une menace pour l'harmonie sacrée du cloître. Son comportement est souvent radical et violent (il a tendance à régler les conflits qui naissent entre lui et ses confrères par une bonne volée de coups), sa personnalité, instable et disharmonieuse, se trouve bouleversée par différentes passions (il mange trop et s'enivre régulièrement) et au moment de son entrée dans les ordres il ne sait pas lire. Le contraste entre l'apparente harmonie de l'espace monastique et le caractère grossier ou - selon Pierre MANEN (2014) et Florian JINGAND (2014) - démesuré du personnage de Guillaume devient, à mesure que le récit progresse, tellement aigu que les moines décident d'éliminer leur confrère.

\section{Le narrateur-juge et son monastère de l'iniquité}

Si la projection mentale de l'abbaye existant au sein de la conscience fictionnelle de la communauté monastique avait été la seule projection mentale doublant la modalité physique du monastère dans le texte, la détermination des moines à chasser Guillaume de leur cosmos aurait été parfaitement légitime. Or, dès l'exposition de l'épopée, l'optique des moines se heurte à une autre optique qui est celle du narrateur, dont la vision du monastère semble être radicalement différente. ${ }^{14}$

Afin de mieux saisir les attributs de cette seconde projection mentale de l'abbaye, arrêtons-nous quelques instants sur la manière dont la catégorie du narrateur se construit dans la chanson de geste analysée. Le narrateur du Moniage Guillaume I est - conformément aux canons de la narration épique médiévale ${ }^{15}$ - un narrateur hétérodiégétique ${ }^{16}$, tantôt à la troisième, tantôt à la première personne du singulier (pour qu' un jongleur, interprétant le texte devant un auditoire empirique, puisse s'emparer de son discours et capter l'attention du public). Par ailleurs, comme Pierre MANEN (2014) le remarque avec beaucoup de pertinence, il s'affirme comme une entité très autoritaire. Au moyen des évaluations axiologiques du comportement des personnages, il intervient dans le récit et acquiert progressivement - et ingénieusement - le plein contrôle du texte et du processus de la communication littéraire. Par ses commentaires souvent ironiques (grâce auxquels il dévoile sa projection mentale de l'espace du monastère), il influence le lecteur ou l'auditeur en offrant à ce dernier la seule interprétation juste des événements narrés et en réduisant ainsi l'ambiguïté potentielle du texte. Il serait même possible de prétendre qu'au sein du monde fictionnel de l'épopée, il représente un porte-parole de Dieu ${ }^{17}$.

\footnotetext{
${ }^{14}$ Observation déjà faite par Pierre MANEN (2014) dont l'étude - il convient de le noter - a largement influencé notre perception de la catégorie du narrateur dans la chanson.

${ }^{15}$ Pour plus de détails sur les mécanismes canoniques de la narration dans les chansons de geste, voir, par exemple, ARAGON (1987).

16 Terme de Gérard Genette désignant un «narrateur absent de l'histoire qu'il raconte » (GENETTE $1972: 252)$.

17 Pour éviter toute confusion éventuelle, il faut noter qu'en faisant mention du «porte-parole de Dieu », nous n'avons point l'intention de rapprocher le narrateur du Moniage Guillaume du narrateur omniscient apprécié par Honoré de Balzac. Nous constatons seulement que le narrateur de la chanson de geste analysée adopte systématiquement un point de vue jugé divin.
} 
Sans le moindre problème, il pénètre dans la conscience des personnages et révèle leurs motivations intrinsèques. D'ailleurs, à ses yeux, Guillaume est un saint ${ }^{18}$. La foi et le comportement du protagoniste sont, selon lui, plaisants à Dieu et dignes d'admiration :

Dont fu Guillaume puis en l'ordene maint jor,

Mout mena sainte vie.

[Nouvelle laisse]

Li quens fu moines, si ot la robe prise ;

Mout volentiers öi le Deu service,

Ne li escape ne messe ne matine,

Tierce ne none ne vespre ne conplie.

(Le Moniage Guillaume 1906 : 8, v. 182-187) ${ }^{19}$

Le monastère, au contraire, s'identifie à une localité non authentique, hostile et violente, partageant un nombre considérable d'attributs spatiaux que Mircea Eliade assigne aux territoires profanes, c'est-à-dire au $\operatorname{chaos}^{20}$. Au regard du narrateur, la haute culture des moines n'est qu' un masque derrière lequel se dissimulent les vices.

Cette position axiologique est perceptible notamment dans les commentaires que le narrateur introduit dans le tissu narratif des épisodes où Guillaume se voit confronté à l'hostilité des autres moines qui « li portent grant envie » (Le Moniage Guillaume 1906 : 8, v. 188), plus particulièrement dans les passages décrivant le rire des moines. Ce dernier, selon André MOISAN (1995), n'a rien à voir avec la tonalité (souvent) burlesque de la chanson de geste étudiée, mais s'affirme comme enfantin, humiliant et vexant, et témoigne d'une incompréhension tragique qui s'établit entre les cloîtriers et leur nouveau confrère. Nous ajouterions même qu'il s'assimile, à certains égards, au rire non maîtrisé que certains érudits médiévaux, à la base de la fameuse assertion «Jésus n'a jamais ri », considéraient comme une émotion inquiétante ${ }^{21}$. Le narrateur le dépeint de manière subtile par exemple dans le passage où Guillaume reçoit la tonsure ${ }^{22}$. Lorsque les moines voient que l'habit offert à Guillaume cadre mal avec la taille démesurée de leur confrère, ils éclatent de rire :

Li quens Guillaume en l'encloistre s'asist.

Courone ot grant, et li abes li fist ;

Et la pelice que il vestir li fist

Courte li fu, et li abes en rist,

\footnotetext{
${ }^{18}$ Le décalage entre l'optique du narrateur et la manière dont Guillaume est perçu par ses confrères est étudié également par Pierre MANEN (2014).

${ }^{19}$ L'assiduité de Guillaume quant à la prière du bréviaire ainsi que son obéissance vis-à-vis de l'abbé sont analysées également par André MoISAN (1995).

${ }^{20}$ Selon Mircea Eliade (1965), les espaces profanes dans leur forme pure présentent les attributs suivants : chaotique, fluide, obscur, illusoire, inconnu et peuplé d'étrangers et d'autres êtres mortifères. ${ }^{21}$ Pour des renseignements supplémentaires sur le phénomène du rire et sa perception au Moyen Âge, voir, par exemple, LE GofF (1989), VALETTE (2012).

${ }^{22}$ Une analyse et interprétation assez détaillée de cette scène peut être trouvée chez André MoISAN (1995).
} 
Et tout li moine s'en rïent autresi,

Cascuns l'esgarde, si se sont esjöi.

Damerdiex le garisse !

(Le Moniage Guillaume 1906 : 7-8, v. 162-168)

Or, la pertinence de l'optique du narrateur et de ses positions axiologiques ne se manifeste pleinement qu'à partir du moment où la communauté monastique commence à préparer le complot contre le protagoniste. Au lieu de chasser Guillaume du monastère par un moyen légitime, l'abbé et la communauté religieuse décident de recourir à la ruse. En espérant que la «brebis galeuse » sera assassinée par une troupe de brigands dans la forêt de Beaucler (li bois de Biaucler) située entre l'abbaye et la ville, l'abbé ordonne à l'ancien guerrier de se rendre en ville pour acheter de la nourriture. Il est certainement inutile de rappeler qu'au Moyen Âge la ruse était considérée comme une pratique diabolique ${ }^{23}$. Cette croyance trouvait ses racines probablement dans le chapitre 3 du livre de la Genèse, où le narrateur biblique constate : «Le serpent était le plus rusé de tous les animaux des champs » (La Bible 2013: 55, Gn 3, 1a). La nature vicieuse de la ruse que l'abbé et la communauté monastique préparent se reflète également dans le langage que les moines commencent à utiliser au moment où la haine et l'envie envers leur nouveau confrère l'emportent sur leur fidélité aux principes de la règle bénédictine. Ils cessent de s'exprimer de manière neutre et noble et passent au registre de la malédiction :

Nostre abes fist une grant dïablie.

(Le Moniage Guillaume 1906 : 9, v. 190)

Qui chi le mist, li cors Dieu le maudie,

Qu'il nos fera tous honte!

(Le Moniage Guillaume 1906 : 9, v. 196-197)

Pour Dieu, sire abes, a vous me vieg clamer

De vostre moine, qui Dex puist mal doner.

Dex le confonde chïens le fist entrer !

Jou port les clés pour vostre bien garder,

As vis dïables soient il commandé !

(Le Moniage Guillaume 1906 : 10, v. 228-232)

\section{Le climax}

Comme en témoignent les arguments mis en valeur aux chapitres précédents, l'optique du narrateur et l'optique des moines s'opposent fermement l'une à l'autre sur le plan axiologique. La question se pose alors de savoir laquelle des deux entités narratives détient, au sein du monde fictionnel du Moniage Guillaume I, la vérité. L'énigme commence à s'éclaircir au moment où Guillaume, rentrant de la ville, pénètre dans la forêt de Beaucler et affronte la troupe de brigands. Le lecteur ou l'auditeur, en envisageant les attributs spatiaux de la forêt et en observant le

\footnotetext{
${ }^{23}$ Pour davantage de renseignements sur les représentations du diable au Moyen Âge et sur le rapport intime entre la ruse et l'esprit malin, voir AsHBY (1979).
} 
comportement des brigands, découvre que le texte établit un effet miroir entre l'espace de la forêt et celui de l'abbaye : la forêt de Beaucler constitue un reflet métaphorique du vrai visage du monastère et de ses résidents. Bien que le Moniage Guillaume I ne contienne presque aucune topographie du bois en question, le lecteur ou l'auditeur sait d'expérience que les forêts incarnent des locus dangereux. Li bois de Biaucler, constitué fort probablement d'une multitude d'arbres effroyablement homogènes, est donc, selon toute vraisemblance, un territoire chaotique, imprévisible, dépourvu de tout point fixe et - étant donné que les rayons du soleil peinent à percer les ramures - plongé dans l'obscurité. En outre, le texte laisse entendre que son sol est couvert de trous. Ce chaos ténébreux fait écho au chaos et à la morale brumeuse qui règnent, selon le narrateur, à l'intérieur du complexe monastique, et la troupe des brigands, qui devient en quelque sorte l'alliée de la communauté monastique, reflète les vices de cette dernière. Tout comme les résidents de l'abbaye de Gênes-sur-Mer, les brigands ne montrent aucune estime envers la religion ni envers la vie humaine, la seule règle gouvernant leur vie communautaire étant celle de la violence et de la convoitise :

\footnotetext{
Ne clerc ne prestre ne vesque ne abé

Ne prisons nous un denier mouneé.

Trop estes rices et d'avoir assasés.

As povres gens dëussiés tout doner.

Et vostre vie dëussiés amender.

Or pensés bien de matines chanter,

Nous penserons de tollir et d'enbler.

(Le Moniage Guillaume 1906 : 20, v. 497-503)
}

Heureusement, grâce à sa clairvoyance et à sa force extraordinaire, Guillaume parvient à éliminer, dans une bagarre courte et énergique, l'ensemble de la troupe de brigands. Néanmoins, lorsqu'il atteint la porte du monastère et demande au portier d'ouvrir le verrou, il apprend que l'abbé refuse de l'admettre à nouveau au sein de la communauté monastique. Ainsi, il prend conscience des intrigues de ses confrères et de la haine que les autres moines ressentent envers lui, et comprend que la rencontre hasardeuse avec les brigands faisait partie du complot préparé par l'abbé. Cette révélation entraîne chez lui une crise de colère violente que nous interprétons (suivant le fil de notre argumentation) comme une métamorphose tangible de l'apogée de la colère du narrateur ou, autrement dit, du «porte-parole de Dieu », colère que le lecteur ou l'auditeur voyait s'accroître à mesure que l'histoire progressait. Cet épisode révélateur marque également le moment du triomphe symbolique de l'optique du narrateur sur celle des moines. La projection mentale de l'espace du monastère conçue par la conscience collective des moines se révèle totalement discréditée, et le lecteur ou l'auditeur ne peut plus douter de la nature fausse et profane de l'espace de l'abbaye. En effet, la sacralité du locus analysé qui semblait résulter des répliques des cloîtriers dans les premières laisses n'était que pure illusion. L'abbaye de Gênes-sur-Mer cesse ainsi de remplir, au sein du continuum spatial du Moniage Guillaume I, la fonction de cosmos et se transforme en chaos. 
C'est pourquoi dans les épisodes finaux du fragment conservé de la chanson de geste, le lecteur ou l'auditeur est témoin d'une (auto-)destruction partielle de l'espace de l'abbaye ${ }^{24}$. Guillaume, saisi de fureur, rompt le verrou de la grande porte et détruit ainsi la frontière qui séparait le cloître du monde extérieur et rendait le complexe monastique, au moins du point de vue architectural, plus rassurant et, par conséquent, plus sacrée 5 . Une fois le verrou brisé, le protagoniste pénètre à l'intérieur et commence à couvrir de coups ses confrères, dont plusieurs, y compris le prieur, se font frapper à mort. Nous nous permettons de citer un morceau plus long de ce passage composé avec une virtuosité stylistique sans pareille, et même avec une pincée de burlesque (surtout par le jeu subtil avec les tournures épiques « ki li vëist» et «qui dont veïst», employées, d'habitude, dans des scènes de bravoure) :

\author{
El cloistre vint dans Guillaume li ber, \\ Si commencha moines a escrïer. \\ Dis en encontre, ne porent escaper, \\ (Ki li vëist a la terre fouler \\ Et de ses poins mout ruistes cols donner !) \\ As caperons les a pris a coubrer : \\ Un en a pris qui ne pot tost errer, \\ Trois tours le tourne, au quart le lait aler, \\ Si roidement le fiert a un piler \\ Qu'andeus les eux li fist del cief voler, \\ Puis li escrie : «A moi venés parler!» \\ Et d'une bote a consüi l'abé, \\ Enmi l'encloistre l'abati tout pasmé. \\ (Li autre moine sont en foies torné.) \\ Qui dont vëist dant Guillaume le ber \\ Parmi l'encloistre et venir et aler, \\ En la quisine et el dortoir entrer ! \\ N'i remest canbre ne face desfremer. \\ (Le Moniage Guillaume 1906 : 33, v. 757-774)
}

La destruction de la modalité physique de l'espace du monastère et la mise à mort de certains de ses résidents est cependant une conséquence logique de l'autodestruction morale qui s'était déjà opérée dans la communauté religieuse auparavant. Guillaume n'est qu'un exécuteur de la colère du narrateur (et, par conséquent, de la colère divine) qui flambait contre les moines depuis qu'ils s'étaient laissés dominer par la haine et l'envie. Toutefois, même après ce climax, la morale des résidents du monastère reste inchangée. Comme l'indique le texte, les moines ne voient pas dans la dévastation de leur espace vital un châtiment divin mais simplement le résultat naturel de la colère non maitrisée du protagoniste. C'est pourquoi, une fois la situation apaisée, ils préservent le statu quo ante bellum : l'abbé proclame : «[...] li mort soient maintenant enterré ; [ja] d'autres moines recoverrons plenté »(Le Moniage Guillaume 1906 : 34, v. 795-796), et le narrateur

\footnotetext{
${ }^{24}$ Cet évènement est abordé également par André Moisan (1995) et Pierre MANEN (2014) qui en donnent néanmoins des interprétations légèrement différentes de la nôtre.

${ }^{25}$ Nous rappelons que selon Mircea EliADE (1965), le caractère clos peut impliquer la sacralité d'un territoire.
} 
n'a d'autre choix que d'ajouter amèrement et laconiquement, à la fin de la laisse XXIX : «Cil qui mort sont furent tost oblié » (Le Moniage Guillaume 1906 : 35, v. 816).

Mais puisque le continuum spatial d'un texte héritier des structures mythiques ${ }^{26}$ comme celui du Moniage Guillaume I ne peut pas rester sans cosmos, à la fin du fragment conservé, le rôle que l'abbaye de Gênes-sur-Mer devait jouer au sein du continuum spatial du texte est revendiqué par un autre espace. Quelque temps après la destruction du monastère, l'ange du Seigneur réapparaît au protagoniste en lui ordonnant de quitter l'abbaye et de se retirer dans un ermitage, une gastine près de Montpellier. Dans les dernières laisses conservées, cette gastine acquiert progressivement le statut de localité sacrée grâce aux attributs qui lui sont assignés par le narrateur (dont le discours, comme nous l'avons observé, est un discours vrai, à la différence du discours des moines) et qui correspondent aux attributs spatiaux du cosmos évoqués par Mircea Eliade. Premièrement, étant donné que le protagoniste restaure la gastine de ses propres mains, cette dernière incarne pour lui un espace ressenti comme sien. Guillaume devient, au sens métaphorique du terme, « ancré dans les murs » de l'ermitage comme les moines de Gênes-sur-Mer étaient «ancrés dans les murs » de leur monastère. Deuxièmement, puisque la gastine embrasse une chapelle avec un autel, Guillaume peut y éprouver l'union mystique avec son Créateur. Enfin, troisièmement, l'ermitage devient pour le protagoniste un espace rassurant : Guillaume entoure son nouvel espace vital de remparts solides qui sépareront l'intérieur du complexe du monde extérieur profane et chaotique, et qui le protégeront contre les éventuelles invasions sarrasines :

Un castelet ot fremé sor le mont

La gist Guillaume pour Sarrasins felons.

(Le Moniage Guillaume 1906 : 37, v. 875-976)

Notons juste que dans l'ultime partie du tissu narratif, le lecteur ou l'auditeur ne retrouve plus aucune mention de l'abbaye de Gênes-sur-Mer. Cette dernière disparaît complètement du continuum spatial.

\section{En guise de conclusion}

Dans les décennies précédentes, le caractère dissonant de la représentation épique de l'abbaye dans le Moniage Guillaume a attiré l'attention de plusieurs médiévistes ${ }^{27}$. La singularité de ce locus paradoxal dominant le monde fictionnel de

\footnotetext{
${ }^{26}$ Pour davantage de renseignements sur les rapports entre la chanson de geste et le mythe, voir BOUTET (1988).

${ }^{27}$ Il convient de noter cependant que la version en vers du Moniage Guillaume nous est parvenue en deux rédactions et que la majorité des études sur l'épopée, y compris celles d'André MoISAN (1995) et de Pierre MANEN (2014), se rapporte à la soi-disant «seconde rédaction» (plus longue, plus complète et probablement plus récente), habituellement désignée par Wilhelm Cloetta ainsi que par d'autres spécialistes comme Moniage Guillaume II. Le Moniage Guillaume I- probablement en raison de son caractère estropié - reçoit systématiquement moins d'attention de la part des experts en poétique médiévale. Pour plus de détails sur les similitudes et les différences entre les deux rédactions, ainsi que sur le Moniage Guillaume II, voir Les deux rédactions en vers du Moniage Guillaume 1906, ClOETTA
} (1911). 
l'épopée repose - comme le prouvent nos analyses - sur les discrépances instaurées entre deux projections mentales doublant, au sein du récit, la modalité physique (ou architecturale) du monastère. Tandis que la projection mentale que la communauté monastique se crée de son cosmos présente des attributs spatiaux d'un locus sacré, le monastère s'identifie aux yeux du narrateur (qui apparaît dans le texte sous la forme d'un «narrateur-juge » ou d'un «porte-parole de Dieu ») à un chaos moral. La «lutte » métaphorique entre l'optique du narrateur et l'optique des moines, autour de laquelle se construit l'ensemble du récit, culmine dans l'effet miroir établi entre l'espace de l'abbaye et celui de l'épouvantable forêt de Beaucler, ainsi que dans la scène d'(auto-)destruction de l'espace du monastère, conséquence de «l'avalanche de perfidie » qui recouvre l'abbaye dans la seconde partie de l'épopée.

Or, pourquoi retrouve-t-on dans un texte du XII ${ }^{\mathrm{e}}$ siècle, siècle baignant dans l'estime envers la religion, une représentation aussi malplaisante d'un complexe monastique $^{28}$ ? À l'époque où fut établi le texte du Moniage Guillaume I tel que nous le connaissons aujourd'hui, la famille religieuse des bénédictins se trouvait en crise. Le populus, mais aussi les autorités ecclésiastiques, reprochaient aux « moines noirs » leur grande richesse et leur réticence vis-à-vis des fidèles laïcs. Ce malaise fit naître plusieurs tendances réformistes, entre autres la fondation de l'ordre cistercien, branche réformée des bénédictins, le retour de l'érémitisme ${ }^{29}$ et l'essor des ordres mendiants au début du XIII ${ }^{e}$ siècle, phénomène socio-culturel que Diarmaid MacCulloch (2010 : 401) dénomme «Pastoral Revolution». Ainsi, suivant les positions adoptées par André MOISAN (1995), nous pourrions voir dans la nature dissonante de la représentation épique de l'abbaye de Gênes-sur-Mer une critique de la sclérose des communautés bénédictines du XII ${ }^{\mathrm{e}}$ siècle, ou un appel littéraire à la réforme du monasticisme occidental...

\section{BIBLIOGRAPHIE}

ANONYME (1906), Les deux rédactions en vers du Moniage Guillaume (tome I), publ. par W. Cloetta, Paris, Société des anciens textes français.

ANONYME (1906), Le Moniage Guillaume, première rédaction, in : Les deux rédactions en vers du Moniage Guillaume (tome I), publ. par W. Cloetta, Paris, Société des anciens textes français, pp. 1-39.

ANONYME (2013), La Bible, traduction officielle liturgique, Paris, Desclée-Mame.

ARAGON Aurora (1987), Statut et fonctions du narrateur dans la chanson de geste, in : Au carrefour des routes d'Europe : la chanson de geste (tome I), Aix-enProvence, Presses universitaires de Provence, pp. 197-222.

AshBy Ginette et al. (1979), Le diable au Moyen Âge: Doctrine, problèmes moraux, représentations, Aix-en-Provence, Presses universitaires de Provence.

\footnotetext{
${ }^{28}$ Le présent paragraphe résume plusieurs idées et faits évoqués chez Gert MeLviLLE (2016), Clifford Hugh LaWrenCE (2013) et Diarmaid MaCCulloch (2010, voir les sous-chapitres Abbots, Warriors and Popes: Cluny's Legacy, Cistercians, Carthusians and Mary et A Pastoral Revolution, Friars and the Fourth Lateran Council).

${ }^{29}$ À mettre en rapport avec le retrait de Guillaume dans la gastine.
} 
BouTET Dominique (1988), Mythe, littérature et société, in : BOUTET Dominique ; HARF-LAnCNER Laurence (éds.), Pour une Mythologie du Moyen Âge, Paris, École Normale Supérieure, pp. 89-97.

Cloetta Wilhelm (1911), Les deux rédactions en vers du Moniage Guillaume (tome II), Paris, Société des anciens textes français.

ČInČUROVÁ Xénia (2004), Epické podoby priestoru, Levoča, Modrý Peter.

DOLEŽEL Lubomír (1998), Heterocosmica: Fiction and Possible Worlds, Baltimore, Johns Hopkins UP.

ELIADE Mircea (1965), Le sacré et le profane, Paris, Gallimard.

GENETTE Gérard (1972), Figures III, Paris, Seuil.

JINGAND Florian (2014), Guillaume et Rainouart : figures du guerrier démesuré (thèse de doctorat), Montpellier, Université Paul Valéry.

LAWRENCE Clifford Hugh (2013), The Friars: The Impact of the Mendicant Orders on Medieval Society, London and New York, I. B. Tauris.

LE GoFf Jacques (1989), Rire au Moyen Âge, Cahiers du Centre de Recherches historiques 3, pp. 1-14.

MACCUlloCh Diarmaid (2010), A History of Christianity, London, Penguin Books.

MANEN Pierre (2014), Guillaume d'Orange dans Le Moniage Guillaume : la force brute de l'autorité, Le Moyen Âge et la Renaissance face aux lois : de la critique à la subversion, Cahiers du CELEC 8, <http://cahiersducelec.univ-stetienne.fr/files/Documents/cahiers_du_celec_8/Pierre\%20Manen\%202.pdf $>$.

MELVILLE Gert (2016), The World of Medieval Monasticism: Its History and Forms of Life, Collegeville, Cistercian Publications.

MoISAN André (1995), L'abbé Henri et ses moines dans le « Moniage Guillaume » et le «Moniage Rainouart» ou la perfidie dans l'état monastique, in : Le clerc au Moyen Âge, Aix-en-Provence, Presses universitaires de Provence, pp. 435447.

LOTMAN Youri (1973), La Structure du texte artistique, Paris, Gallimard.

TKÁČ Martin (2012), Eucharistia - vrchol iniciácie, in : ANDREJČÁKOVÁ Petra et al. (éds.), “In pluribus unum - jednota v mnohosti”: zborník príspevkov z medzinárodnej konferencie doktorandov a mladých vedeckých pracovníkov teológie, Prešov, Prešovská univerzita v Prešove, Gréckokatolícka teologická fakulta, pp. 365-377.

VALETTE Jean-René (2012), Le rire et le corps : éléments d'esthétique médiévale $\left(\mathrm{XII}^{\mathrm{e}}-\mathrm{XIII}^{\mathrm{e}}\right.$ siècle), in : VAILlANT Alain (éd.), Esthétique du rire, Nanterre, Presses universitaires de Paris Ouest, pp. 21-45. 